\title{
Una aproximación al Estado del Arte Ventana de Observación: Legislación Internacional del Control Fiscal, en el marco de la evaluación integral las Contralorías Territoriales
}

\author{
An approach to the State of the Art Observation Window: International \\ Legislation of Fiscal Control, within the framework of the comprehensive \\ evaluation of the Territorial Comptrollers
}

DOI: 10.13140/RG.2.2.28011.36645

Betthy Constanza Lizarazo Araque

Luz Angela Valenzuela Acosta

\section{Resumen}

La contribución a la construcción del Estado del Arte desde la ventana de observación: Legislación Internacional del Control Fiscal, en el marco de la evaluación integral las Contralorías Territoriales, demanda procesos de selección, caracterización e interpretación del conjunto de disposiciones y orientaciones normativas aplicables tanto a nivel local, como global, sobre la gestión de los recursos públicos y las responsabilidades de los Estados, referida a las buenas prácticas de todos los actores que intervienen en el proceso, a partir de la aplicación del Derecho Internacional que proporciona el marco jurídico, apoyado por el papel que desempeñan los Organismos Internacionales en la formulación de políticas públicas globales, sobre el proceso de desarrollo integral de las naciones y reconocimiento de los derechos humanos.

\section{Summary}

The contribution to the construction of the State of the Art from the observation window: International Legislation of Fiscal Control, within the framework of the comprehensive evaluation of the Territorial Comptrollers, demands selection processes, characterization and interpretation of the set of provisions and normative guidelines applicable to both local, as well as global, on the management of public resources and the responsibilities of the States, referring to the good practices of all the actors involved in the process, based on the application of International Law that provides the legal framework, supported in the role played by International Organizations in the formulation of global public policies, on the process of integral development of nations and recognition of human rights.

Palabras claves: Política pública, Control fiscal, Legislación internacional, Contralorías territoriales, Corrupción, Participación ciudadana, Gasto público, Derechos humanos

Key words: Public policy, Fiscal control, International legislation, Territorial comptrollers, Corruption, Citizen participation, Public spending, Human rights 


\section{Introducción}

En primer lugar, es pertinente anotar que el Estado del Arte es una investigación que pretende informar sobre construcciones de bases de datos que apoyan un diagnóstico, y, en consecuencia, corresponde a un concepto más amplio de lo que se conoce como revisión documental (que es parte sustancial de un estado del arte), dando cuenta del avance de las investigaciones sobre un tema concreto. Los Estados del Arte en las ciencias sociales, constituyen un acercamiento a la apropiación de la realidad como tal, estableciendo una propuesta interpretativa inicial de otras investigaciones ya realizadas. (Jiménez, 2004). Como lo expone Ragnhild Guevara Patiño, citando a Uribe (2002) en su artículo denominado: El estado del arte en la investigación: ¿análisis de los conocimientos acumulados o indagación por nuevos sentidos?:

...el estado del arte es una investigación sobre la producción investigativa de un determinado fenómeno. Este permite develar la dinámica a partir de la cual se ha desarrollado la descripción, explicación o comprensión del fenómeno en estudio y la construcción de conocimientos sobre el saber acumulado. (Guevara, 2016, pág. 169).

A partir de la gnoseología desde sus tres vertientes: proposicional (saber que), práctico (saber cómo) y directo (conocer) (Robles, 1949), se ha realizado este acercamiento a la construcción del Estado del Arte de la ventana de observación referida, permitiendo la identificación del camino para la indagación de la información requerida, dada la gran cantidad de literatura disponible sobre la temática propuesta. Para seleccionar las publicaciones relacionadas más adelante, se realizó un proceso de búsqueda desde los portales de plataformas como Scimago, LaReferencia, y Google Académico. La metodología utilizada durante el proceso es de tipo cualitativo e interpretativo, que permitió determinar la selección de la muestra documental.

El primer paso de este proceso consistió en la construcción de una base de datos en Excel, que después de haber sido depurada, está conformada por 39 publicaciones de diferente tipo: Trabajos de investigación, tesis de pregrado y postgrado, artículos de revista, manuales, y otros; publicados tanto a nivel local, como internacional entre los años 1977 y 2019. Vale la pena anotar, que probablemente se han escapado publicaciones muy valiosas al respecto, pero que seguramente serán retomadas en un futuro próximo. El siguiente paso, fue el diseño de los mapas conceptuales correspondientes, en los que se esquematizaron y sintetizaron las ideas principales expuestas por cada uno de los autores.

Finalmente, y como se muestra a continuación, se elaboraron las reseñas pertinentes para cada una de las publicaciones.

\section{Consolidación del trabajo de investigación}

La ventana de observación denominada Legislación Internacional del Control Fiscal en el marco de la evaluación integral las Contralorías Territoriales, tiene como 
objetivo principal realizar una aproximación significativa a la ampliación de resultados de investigación, monitoreo y seguimiento permanente, a partir de la recopilación y análisis de documentos disponibles y emitidos por fuentes idóneas, con el fin de visibilizar la democratización del conocimiento en esta materia.

Con el trabajo realizado, se pretende un acercamiento significativo a las diversas dinámicas internacionales sociopolíticas, económicas y jurídicas contemporáneas, que como sabemos, cada vez son más globales, problemáticas y cambiantes, por cuanto generan fenómenos y situaciones críticas desde diferentes contextos gubernamentales, interinstitucionales, académicos y sociales. Así las cosas, se relaciona una sinopsis sobre las 39 reseñas enunciadas, que incluyen temáticas como:

- Un breve paso por el proceso histórico del control fiscal desde el descubrimiento, la colonia y la época de la república, entre las que se cuentan la Misión Kemmer y las reformas realizadas antes de 1975, hasta la reforma de la Constitución Política de Colombia en 1991.

- Una mirada a las prácticas anticorrupción de las organizaciones adheridas al pacto global en Colombia, donde los elementos de gestión para combatir la corrupción se resumen en el compromiso y unidad de esfuerzo del Estado, las empresas (tanto públicas como privadas) y la sociedad civil. En este proceso, se evidencia la corrupción como problema jurídico y como una condición sociológica-moral. (García, 2012). Este flagelo se considera también como un problema sociopolítico y económico, afectando no sólo los negocios internacionales, sino, la legitimidad y la confianza de los Estados, requiriendo una pronta solución, desde las fuentes del Derecho Internacional Público. Al respecto, se incluyen principios, objetivos y normas que se acogieron en la Convención de las Naciones Unidas contra la Corrupción.

- Así mismo, la Organización Internacional de Entidades Fiscalizadoras Superiores (INTOSAI), contribuye al fortalecimiento de la fiscalización de recursos públicos, en busca de la homologación de normas y procedimientos, apoyando desde diferentes programas y seminarios la lucha contra la corrupción y ofreciendo el marco de los principios esenciales para la correcta fiscalización del sector público desde las Entidades Fiscalizadoras Superiores (EFS), con el fin de que sean adoptados, publicados y aplicados desde las instancias correspondientes. (INTOSAI, 1996). Este marco normativo de directrices, se circunscribe en las diferentes ISSAI, entre las que se cuentan: ISSAI 1: Declaración de Lima, ISSAI 10: Declaración de México sobre la Independencia de las EFS, ISSAI 12: EI Valor y Beneficio de las Entidades Fiscalizadoras Superiores - marcando la diferencia en la vida de los ciudadanos, ISSAI 20: Principios de transparencia y rendición de cuentas, ISSAI 50: Principios de las Actividades Jurisdiccionales de las EFS, ISSAI 100: Principios Fundamentales de Auditoría del Sector Público, ISSAI 30: Código de ética, ISSAI 40: Control de Calidad para la EFS, ISSAI 200: Principios Fundamentales de la Auditoría Financiera, ISSAI 300: Principios Fundamentales de la Auditoría de Desempeño, e ISSAI 400: Principios Fundamentales de la Auditoría de Cumplimiento. 
- La evaluación de la preparación para la implementación de la agenda 2030 y los objetivos de desarrollo sostenible en Colombia realizada por la Contraloría General. (Contraloría, 2018).

- La responsabilidad del Estado y el criterio de sostenibilidad fiscal, que establece que ningún derecho constitucional, puede ser negado en aras de la sostenibilidad fiscal. (Arias, 2017).

- La Autonomía fiscal de las entidades territoriales en Colombia, como una propuesta legislativa de la autonomía tributaria de los entes territoriales, sobre los cuales se construye una tesis de la inexistencia de su autonomía en la práctica. (Holguin, Montoya, \& Paez, 2014).

- Como pasar de un principio de ejecución presupuestal, a un principio de eficacia en el manejo del patrimonio público, teniendo en cuenta que a partir de las recomendaciones de la OCDE, el ámbito internacional reclama mayor flexibilización del gasto público, a partir de una planeación de largo plazo, de tal forma que se logren los objetivos de eficiencia y eficacia del sector público (Garcés, Bautista, \& Díaz, 2019).

- El Impacto del control fiscal, referido a la optimización de los recursos públicos (físicos, humanos, financieros y el medio ambiente), respecto de las semejanzas, diferencias, atributos característicos y las causas identificables entre la regulación de los distintos países. (Cabrera, 2014).

- Algunas normas, reformas, logros y recomendaciones, respecto de la contratación pública, tanto centralizada, como descentralizada, considerando la lucha anticorrupción a nivel internacional, desde las esferas de acción local e internacional, que por supuesto, incluyen la veeduría ciudadana, teniendo en cuenta la visión de los derechos humanos, los derechos fundamentales y sus garantías, con la relevancia que implica la ética pública y los valores asociados a la moral, como principales instrumentos de lucha contra la corrupción, y que corresponden al eje principal para el desarrollo de un buen trabajo de auditoría.

- De otra parte, y con el fin de que se ratifiquen los compromisos preestablecidos, con la contribución de organismos multilaterales e interinstitucionales, tales como la OCDE, el BM, el FMI, Naciones Unidas y la CEPAL, entre otros, se presentan diferentes disposiciones para la globalización de la ética, respeto de los bienes públicos globales y los derechos públicos, económicos y sociales, para la búsqueda universal del respeto por la vida y la dignidad humana. (Artigas, 2001).

- Además de todo lo anterior, se presentan varias posiciones críticas de algunos autores, respecto de la proliferación de la normatividad vigente que en algunos casos, desorienta la interpretación de los funcionarios públicos en la aplicación correspondiente, por cuanto se aduce que, las reglas de la contratación pública no son claras, y presentan múltiples incoherencias contribuyendo a la ineficiencia e ineficacia, en el ejercicio de la gerencia pública. 


\section{Conclusiones:}

El aporte al conocimiento, a partir del reservorio de la información contenida en los documentos reseñados, alimenta de manera significativa el Estado del Arte para la ventana de observación "Legislación Internacional del Control Fiscal, en el marco de la evaluación integral las Contralorías Territoriales", contextualizando e integrando temas desde diferentes perspectivas sobre la legislación, la política, la sociología, y la economía (entre otros); nutriendo el espectro disponible de consulta del Observatorio de Política Pública del Control fiscal de la AGR.

Las normas, reformas, logros y recomendaciones respecto de la contratación pública (centralizada y descentralizada) consideran la lucha contra la corrupción tanto a nivel nacional, como internacional, incluyendo la significación de la veeduría ciudadana, teniendo en cuenta la visión de los derechos humanos y sus garantías, con la relevancia que implica la ética pública y los valores asociados a la moral, como principales instrumentos de lucha contra la corrupción, y que corresponden al eje principal para el desarrollo de un buen trabajo de auditoría.

El análisis de los diferentes temas referidos a la gestión transparente de los recursos públicos, indudablemente constituyen un esfuerzo mancomunado entre las organizaciones internacionales, las instituciones, el Estado y de la sociedad civil en general, para combatir el flagelo de la corrupción, que ha debilitado significativamente la capacidad gubernamental, las instituciones y la inversión social, fracturando el Estado de Derecho y la democracia, debilitando los principios éticos y morales, y desprestigiando la autoridad, reflejada en la injusticia que mancilla la dignidad humana.

La manifiesta indignación generalizada en la sociedad global, reclama un cambio urgente en la mentalidad y exhorta una conducta que promueva la desaparición radical de las prácticas delictivas sobre el erario; concluyendo que la corrupción debe ser tratada y sancionada ejemplarmente.

El fortalecimiento de la educación integral sobre el respeto y valoración de los bienes públicos, contribuirá a la revitalización de valores morales y cívicos para lograr una sociedad más equitativa y justa, que propenda por el crecimiento y consolidación de las naciones. Este propósito, debe trascender a la idoneidad de funcionarios públicos más eficientes y eficaces, que desarrollen una gestión coherente, transparente y oportuna, respecto de los procesos de vigilancia y control sobre los recursos públicos, en todas sus instancias y en las diferentes instituciones. 


\section{Referencias:}

Arias, J. C. (2017). La responsabilidad del Estado y el criterio de sostenibilidad fiscal. La responsabilidad del Estado y el criterio de sostenibilidad fiscal. Bogotá, D.C., Colombia.

Artigas, C. (2001). El aporte de Naciones Unidas a la globalización de la ética. Revisión de algunas oportunidades. Santiago de Chile: Unidad Conjunta CEPAL/OACDH.

Cabrera, G. D. (2014). Impacto del control fiscal ambiental en la optimización de los recursos naturales y el ambiente. Impacto del control fiscal ambiental en la optimización de los recursos naturales y el ambiente. Bogotá, D.C., Colombia.

Contraloría, G. d. (2018). Evaluación de la preparación para la implementación de la Agenda 2030 y los objetivos de desarrollo sostenible en Colombia. Bogotá, D.C.: CGR.

Garcés, R. S., Bautista, S. B., \& Díaz, B. J. (2019). De un principio de ejecución presupuestal a un principio de eficacia en el manejo del patrimonio público. De un principio de ejecución presupuestal a un principio de eficacia en el manejo del patrimonio público. Bogotá, D.C, Colombia.

García, E. (2012). ¿ES COLOMBIA UN ESTADO CORRUPTO? LA CORRUPCIÓN COMO PROBLEMA JURÍDICO Y COMO ESTADO SOCIOLÓGICO-MORAL. Vniversitas, 187-217.

Guevara, P. R. (2016). El estado del arte en la investigación: ¿análisis de los conocimientos acumulados o indagación por nuevos sentidos? Folios, 165179.

Holguin, R. Y., Montoya, T. J., \& Paez, Y. S. (2014). Autonomía fiscal de las entidades territoriales en Colombia. Autonomía fiscal de las entidades territoriales en Colombia. Bogotá, D.C., Colombia.

INTOSAI. (1996). EL PAPEL DE LAS EFS EN LA LUCHA CONTRA LA CORRUPCIÓN Y MALA GESTIÓN. Viena: Naciones Unidas.

Jiménez, B. A. (2004). El estado del arte en la investigación en las ciencias sociales. En A. Jiménez Becerra, La práctica investigativa en ciencias sociales (págs. 29-42). Bogotá, D.C.: UPN, Universidad Pedagógica Nacional.

Robles, O. (1949). Gnoseología fundamental . Actas del Primer Congreso Nacional de Filosofía, (págs. 1190 - 1194). Mendoza, Argentina. 